\title{
Article
}

\section{3'-UTR Polymorphisms in Thymidylate Synthase with Colorectal Cancer Prevalence and Prognosis}

\author{
Young-Joo Jeon ${ }^{1,+}{ }^{\dagger}$, Sung-Hwan Cho ${ }^{1,+}{ }^{\dagger}$, Eo-Jin Kim ${ }^{2,+}{ }^{\oplus}$, Chang-Soo Ryu ${ }^{1}$, Han-Sung Park ${ }^{1}$, Jong-Woo Kim ${ }^{3, *}$, \\ Jeong-Yong Lee ${ }^{1}$, Hui-Jeong An ${ }^{1,4}$ and Nam-Keun Kim ${ }^{1, *}$ (D)
}

1 Department of Biomedical Science, College of Life Science, CHA University, Seongnam 13488, Korea; white0885@naver.com (Y.-J.J.); arana006@naver.com (S.-H.C.); regis2040@nate.com (C.-S.R.); hahnsung@naver.com (H.-S.P.); smilee3625@naver.com (J.-Y.L.); tody2209@naver.com (H.-J.A.)

2 Division of Hematology/Oncology, Department of Internal Medicine, Kangbuk Samsung Hospital, Sungkyunkwan University School of Medicine, Seoul 06351, Korea; eojin1.kim@samsung.com

3 CHA Bundang Medical Center, Department of Surgery, CHA University, Seongnam 13496, Korea

4 College of Life Science, Gangneung-Wonju National University, 7 Jukheon-gil, Gangneung 25457, Korea

* Correspondence: kjw@chamc.co.kr (J.-W.K.); nkkim@cha.ac.kr (N.-K.K.); Tel.: +82-3-1780-5250 (J.-W.K.); +82-3-1881-7137 (N.-K.K.); Fax: +82-3-1780-5259 (J.-W.K.); +82-3-1881-7249 (N.-K.K.)

+ These authors contributed equally to this study.

check for

updates

Citation: Jeon, Y.-J.; Cho, S.-H.; Kim,

E.-J.; Ryu, C.-S.; Park, H.-S.; Kim,

J.-W.; Lee, J.-Y.; An, H.-J.; Kim, N.-K.

3'-UTR Polymorphisms in

Thymidylate Synthase with

Colorectal Cancer Prevalence and

Prognosis. J. Pers. Med. 2021, 11, 537.

https://doi.org/10.3390/jpm11060537

Academic Editor: Lisa Salvatore

Received: 27 April 2021

Accepted: 4 June 2021

Published: 9 June 2021

Publisher's Note: MDPI stays neutral with regard to jurisdictional claims in published maps and institutional affiliations.

Copyright: (C) 2021 by the authors. Licensee MDPI, Basel, Switzerland. This article is an open access article distributed under the terms and conditions of the Creative Commons Attribution (CC BY) license (https:/ / creativecommons.org/licenses/by/ $4.0 /)$.

\begin{abstract}
Colorectal cancer (CRC) is the third most common type of cancer and the second leading cause of cancer-related mortality in Western countries. Polymorphisms in one-carbon metabolism and angiogenesis-related genes have been shown to play important roles in tumor development, progression, and metastasis for many cancers, including CRC. Moreover, recent studies have reported that polymorphisms in specific microRNA (miRNA)-binding regions, which are located in the $3^{\prime}$ untranslated region (UTR) of miRNA-regulated genes, are present in a variety of cancers. Here, we investigated the association between two thymidylate synthase (TYMS or TS) 3'-UTR polymorphisms, 1100T >C [rs699517] and 1170A > G [rs2790], and CRC susceptibility and progression in Korean patients. A total of 450 CRC patients and 400 healthy controls were enrolled in this study, and genotyping at the TS locus was performed by polymerase chain reaction-restriction fragment length polymorphism (PCR-RFLP) or TaqMan allelic discrimination assays. We found that TS 1170A >G genotypes, as well as the TS 1100T-1170G and 1100C-1170A haplotypes, are strongly associated with CRC. The TS 1100TC+CC type was associated with a poor survival (OS and RFS) rate. In addition, levels of the TS 1100C and TS 1170G allele were found to be significantly increased in CRC tissue. Our study provides the first evidence for 3'-UTR variants in TS genes as potential biomarkers of CRC prognosis and prevention.
\end{abstract}

Keywords: thymidylate synthase; 3'-UTR; miRNA polymorphism; colorectal cancer; prevalence; prognosis

\section{Introduction}

Colorectal cancer (CRC) is the third most common cancer worldwide, with 1,849,518 new cases diagnosed per year and representing $10.2 \%$ of all new cancer cases. This disease is also the second most common cancer in women, with 823,303 cases per year, which represents $9.5 \%$ of total cancer cases in women [1]. The geographical incidence of CRC varies, but patterns are similar in men and women, and approximately $55 \%$ of CRC cases occur in more developed regions [2]. Several intrinsic factors, such as age, sex, diabetes mellitus, obesity, and inflammatory bowel disease, as well as extrinsic variables, including cigarette smoking, inadequate fiber intake, high alcohol consumption, red meat consumption, and a high-fat diet, are associated with increased CRC risk [3-5]. Thus, CRC susceptibility appears to be influenced by both genetic and environmental factors.

Recently, one-carbon (1C) metabolism has received considerable attention for its role in cancer malignancies, and consequently, therapeutic agents targeting this pathway 
are increasingly being developed as anticancer agents. Several genes are involved in $1 \mathrm{C}$ metabolism, including methylenetetrahydrofolate reductase (MTHFR), 5-methyltetrahydro folate-homocysteine methyltransferase (MTR), 5-methyltetrahydrofolate-homocysteine methyltransferase reductase (MTRR), cytoplasmic serine hydroxymethyltransferase (cSHMT), dihydrofolate reductase (DHFR), betaine-homocysteine S-methyltransferase (BHMT), and thymidylate synthase (TYMS or TS) [6]. The TS protein catalyzes the conversion of deoxyuridine monophosphate (dUMP) to deoxythymidine monophosphate (dTMP). This process is essential for the production of thymine, a type of nucleotide required for DNA synthesis and repair $[7,8]$. Once synthesized, dTMP is then metabolized intracellularly to dTTP (the triphosphate form), an essential precursor for DNA biosynthesis. This reaction is also critical, as it maintains the essential metabolic requirements needed for cellular proliferation and growth. Because of its key role in DNA replication, human TS is an anticancer drug target $[9,10]$. In particular, this protein is targeted by the widely used anticancer agent 5-FU, which is active against solid tumors, such as breast, head and neck, and colon cancers. Notably, elevated TS levels are correlated with a poorer prognosis, and increased amounts of TS in tumors are associated with resistance to 5-FU chemotherapy [11]. Therefore, TS levels have been suggested as a prognostic factor for CRC survival $[12,13]$ and the response of tumor cells to 5-FU therapy.

A number of studies have investigated possible roles for polymorphisms in several 1C metabolism genes in cancer, including those in TS, which may affect CRC susceptibility, disease progression, and TS-targeted chemotherapy [14-18]. Among these are two well-studied TS polymorphisms: (1) TS enhancer region (TSER) 2R/3R and (2) TS 1494del6 [19-21]. One previous study showed that CRC tumor tissue containing the triple repeat (3R) in the TS enhancer exhibits 4-fold higher TS mRNA levels than CRC tumor tissue from patients who carry the $2 \mathrm{R}$ variant $(p<0.004)$ [22]. This polymorphism is of clinical significance, as greater TS enzyme activity is observed in cancer cells containing the triple repeat than for those with the double repeat $[19,23]$. Another study reported that in 5-FU-treated patients, the presence of a homozygous 6-bp deletion $(-6 \mathrm{bp} /-6 \mathrm{bp})$ in the TS $3^{\prime}$-untranslated region (UTR), which decreases TS mRNA stability and is associated with decreased TS expression in vivo, is correlated with increased treatment efficacy in at least $20 \%$ of the study population [24]. In particular, despite the large amount of data showing the association between these polymorphisms and CRCs, the genetic mechanisms underpinning the role of most TS polymorphisms in CRCs are unclear. [25]. Recent studies have revealed that the $3^{\prime}$-UTR region serves as the binding site for micro RNAs (miRNAs), small non-coding RNAs that function to promote post-transcriptional silencing of target gene expression. Notably, there is also evidence that polymorphisms in the $3^{\prime}$-UTR of a number genes, which are predicted to alter binding sites for specific miRNAs, may be involved in various cancers [26-29]. However, the function of such variants in the $3^{\prime}$-UTR of TS remains unclear.

In the present study, we performed a database search and identified two single nucleotide polymorphisms (SNPs) in the TS 3'-UTR: TS 1100T>C (rs699517) and TS 1170A $>$ G (rs2790). These SNPs are found at minor allele frequencies of $>5 \%$ in the Asian population; however, it is not known whether they display any genetic associations with CRC or if there is variation in TS expression as a function of these $3^{\prime}$-UTR polymorphisms. Therefore, in this study, we investigated whether these polymorphisms in the TS $3^{\prime}$-UTR correlate with CRC development and TS mRNA expression levels.

\section{Materials and Methods}

\subsection{Ethics Statement}

The study protocol was approved (IRB No. 2009-08-077) by the Institutional Review Board of CHA Bundang Medical Center, Seongnam, South Korea. All study subjects provided written informed consent, and all of the methods applied in this study were carried out in accordance with the approved guidelines. 


\subsection{Subjects}

We conducted a case-control study of 850 individuals, including 450 CRC patients and 400 healthy controls. A total of 450 patients diagnosed with CRC at CHA Bundang Medical Center (Seongnam, South Korea) were enrolled from June 1996 to January 2010. We included only CRC patients who had undergone surgical resection with a curative intent and who had histologically confirmed adenocarcinoma. Within the CRC cohort, 155 consecutive patients with proximal colon cancer (i.e., from the cecum to the splenic flexure), 101 consecutive patients with distal colon cancer (i.e., descending and sigmoid colon), 186 consecutive patients with rectal cancer, 4 consecutive patients with mixed colon cancer, and 4 consecutive patients with unclassified CRC underwent primary surgery. We retrospectively obtained information on the date of diagnosis, pathological stage, relapse, and death. Tumor staging of CRCs was performed according to the sixth edition of the American Joint Committee on Cancer (AJCC) Cancer Staging Manual.

The control group consisted of 400 individuals who were randomly selected following a health screening. We excluded patients with a history of thrombotic diseases or cancer. Patients with a high baseline blood pressure (systolic $\geq 140 \mathrm{~mm} \mathrm{Hg}$ or diastolic $\geq 90 \mathrm{mmHg}$ ) on more than one occasion or with a history of antihypertensive medication were classified as having hypertension (HTN). Patients with high fasting plasma glucose $(\geq 126 \mathrm{mg} / \mathrm{dL})$, who took oral hypoglycemic agents, or with a history of insulin treatment were classified as having diabetes mellitus (DM). All study subjects were ethnic Koreans and provided written informed consent to participate.

\subsection{Phenotype Measurements}

Anthropometric measurements included the body mass index (BMI). Systolic and diastolic blood pressures were measured in the seated position after $10 \mathrm{~min}$ of rest. For measurements of physiological parameters, $3 \mathrm{~mL}$ blood samples were obtained after overnight fasting. Plasma glucose levels were measured in duplicate by the hexokinase method, adapted to an automated analyzer (TBA 200FR NEO, Toshiba Medical Systems, Tokyo, Japan). Levels of high-density lipoprotein cholesterol (HDL-C) were determined by enzymatic colorimetric methods, using commercial reagent sets (Toshiba Medical Systems). Plasma homocysteine (Hcy) concentrations were measured by fluorescent polarizing immunoassay (FPIA) with the IMx automated immunohistochemistry system (Abbott Laboratories, Chicago, IL, USA). Plasma concentrations of fatty acids (FA) were determined using a radioassay kit (ACS:180, Bayer, Tarrytown, NY, USA).

\subsection{Genotyping}

DNA was extracted from leukocytes using the G-DEX II Genomic DNA Extraction Kit (Intron Biotechnology, Seongnam, Korea), according to the manufacturer instructions. Genotyping for TS 1100T $>C$ and TS 1170A $>$ G was performed by polymerase chain reactionrestriction fragment length polymorphism (PCR-RFLP) assays. Primers and TaqMan probes were designed using Primer Express Software (v. 2.0) and synthesized by Applied Biosystems (Foster City, CA, USA), with the FAM and JOE reporter dyes. To detect the TS $1100 \mathrm{~T}>\mathrm{C}$ and $1170 \mathrm{~A}>\mathrm{G}$ genotypes, $\mathrm{PCR}$ amplification was performed with forward (5'-GGT ACA ATC CGC ATC CAA CTA TTA-3') and reverse (5'-CTG ATA GGT CAC GGA CAG ATT T- $3^{\prime}$ ) primers, producing a fragment of $170 \mathrm{bp}$. PCR products were digested with 5U BanII (TS 1100T>C) or 3U MboII (TS 1170A >G) for $16 \mathrm{~h}$ at $37^{\circ} \mathrm{C}$.

For $1100 \mathrm{~T}>\mathrm{C}$, the TT genotype was identified by restriction products of $170 \mathrm{bp}$; the TC genotype was identified by products of $170 \mathrm{bp}, 108 \mathrm{bp}$, and $62 \mathrm{bp}$; and the CC genotype was identified by products of $108 \mathrm{bp}$ and $62 \mathrm{bp}$. For $1170 \mathrm{~A}>\mathrm{G}$, the AA genotype was identified by restriction products of $170 \mathrm{bp}$; the AG genotype was identified by products of $170 \mathrm{bp}, 142 \mathrm{bp}$, and $28 \mathrm{bp}$; and the GG genotype was identified by products of $142 \mathrm{bp}$ and 28 bp products. For each polymorphism, 30\% of the PCR assays were randomly selected and repeated, followed by DNA sequencing with an ABI 3730xl DNA Analyzer (Applied 
Biosystems), to validate the experimental findings. The concordance for quality control samples was $100 \%$.

\subsection{Quantitative Reverse Transcription-PCR}

Total RNA for quantitative reverse transcription (qRT)-PCR was extracted from 94 colorectal tissues (47 tumor and 47 tumor-adjacent tissues) of CRC patients using TRIzol reagent (Invitrogen, Thermo Fisher Scientific, Waltham, MA, USA) according to the manufacturer instructions. Synthesis of cDNA from total RNA was performed with the SuperScript III First-Strand Synthesis System (Invitrogen). TS expression levels in tissue were calculated using a comparative $\mathrm{CT}\left(2^{-\Delta \Delta C \mathrm{~T}}\right)$ method, with the $18 \mathrm{~s}$ rRNA gene serving as an internal control. Primer sequences for amplification were as follows: 18s rRNA: forward 5'-AAC TTT CGA TGG TAG TCG CCG-3' and reverse 5'-CCT TGG ATG TGG TAG CCG TTT-3'; TS: forward $5^{\prime}$-CAA CCC TGA CGA CAG AAG AA- $3^{\prime}$ and reverse 5'-GCT CAC TGT TCA CCA CAT AGA-3'.

\subsection{Statistical Analysis}

To analyze baseline characteristics and compare patient and control data, chi-square tests were used for categorical data, and Student's t-tests were used for continuous data. Associations between TS $3^{\prime}$-UTR polymorphisms and CRC incidence were calculated using adjusted odds ratios (AORs) and 95\% confidence intervals (95\% CIs) from multivariate logistic regression analysis, adjusted for age, gender, HTN, DM, BMI, and HDL-C. The variables HTN, DM, BMI, and HDL-C were selected for adjustment, since the risk factors for metabolic syndrome are closely associated with CRC [30]. Allelic gene-gene interaction analysis was performed with the open-source multidimensional reduction (MDR) software package (v. 2.0) available from www.epistasis.org. The MDR method consists of two main steps [31]. First, the best combination of multi-factors is selected, and second, genotype combinations are classified into high- and low-risk groups [31]. For a detailed discussion see Ritchie et al. [32] and Moore and William [33]. We constructed all possible allelic combinations by MDR analysis to identify combinations with strong synergy. Allelic combinations and haplotypes for multiple loci were estimated using the expectation-maximization algorithm with SNPAlyze (v. 5.1; DYNACOM Co, Ltd., Yokohama, Japan), and those having frequencies $<1 \%$ were excluded from statistical analysis. Cox regression models were used to analyze the independent prognostic importance of various markers, and the results were adjusted for age, gender, tumor differentiation, tumor site, chemotherapy, and cancer stage. These calculations excluded 100 CRC patients for whom an insufficient medical history was obtained. Overall survival (OS) was defined as the time from surgery until death or the last follow-up, and relapse-free survival (RFS) was defined as the time from surgery until cancer recurrence or the last follow-up. Adjusted hazard ratios (AHRs) are presented with a 95\% CI. Participants were followed for a median of 34 months (range, 4-173 months). The estimated 3-year OS and RFS rates for all patients were $82.6 \%$ and $81.7 \%$, respectively. Analyses were performed using GraphPad Prism v. 4.0 (GraphPad Software Inc., San Diego, CA, USA) and MedCalc v. 12.7.1.0 (MedCalc Software, Mariakerke, Belgium).

\section{Results}

\subsection{Patient Characteristics}

We collected blood samples and clinical data from 450 CRC patients, including 212 men and 238 women. The mean age was 62.5 years (standard deviation $(\mathrm{SD})=12.29$ ) (Table 1). A total of 260 patients (57.8\%) had colon cancer, and 186 patients $(41.3 \%)$ had rectal cancer. Pathological staging after curative resection was as follows: $42(9.3 \%)$ patients with tumor node metastasis (TNM) stage I, 189 (42.0\%) patients with stage II, $173(38.4 \%)$ patients with stage III, and $46(10.2 \%)$ patients with stage IV. CRC patients and control subjects showed no differences in age or gender ( $p=0.162$ and $p=0.177$, respectively). However, we found that CRC patients were significantly more likely to have HTN $(p<0.001)$, 
$\operatorname{DM}(p<0.001)$, low HDL-C levels $(p<0.001)$, and decreased FA levels $(p=0.043)$, relative to healthy controls.

Table 1. Baseline characteristics of colorectal cancer patients and control subjects.

\begin{tabular}{|c|c|c|c|c|c|c|c|}
\hline Characteristics & Control & CRC & $p$ & Colon & $p$ & Rectum & $p$ \\
\hline$N$ & 400 & 450 & & 260 & & 186 & \\
\hline Age: years (mean $\pm S D$ ) & $60.89 \pm 11.72$ & $62.05 \pm 12.29$ & 0.162 & $61.67 \pm 12.86$ & 0.426 & $62.33 \pm 11.46$ & 0.165 \\
\hline Gender male: $n(\%)$ & $170(42.5)$ & $212(47.1)$ & 0.177 & $122(46.9)$ & 0.299 & $88(47.3)$ & 0.316 \\
\hline Hypertension: $n(\%)$ & $157(39.3)$ & $279(62.0)$ & $<0.001$ & 155 (59.6) & $<0.001$ & $120(64.5)$ & $<0.001$ \\
\hline Diabetes mellitus: $n(\%)$ & $166(41.5)$ & $253(56.2)$ & $<0.001$ & $147(56.5)$ & $<0.001$ & $104(55.9)$ & 0.002 \\
\hline $\mathrm{BMI} \geq 25 \mathrm{~kg} / \mathrm{m}^{2}: n(\%)$ & $93(23.3)$ & $116(25.8)$ & 0.393 & $63(24.2)$ & 0.845 & $51(27.4)$ & 0.323 \\
\hline $\begin{array}{l}\text { HDL-C }<40 \text { (male) or } 50 \\
\text { (female) mg/dL: } n(\%)\end{array}$ & $78(19.5)$ & $197(43.8)$ & $<0.001$ & $111(42.7)$ & $<0.001$ & $84(45.2)$ & $<0.001$ \\
\hline Homocysteine: $\mu \mathrm{mol} / \mathrm{L}(n)$ & $\begin{array}{l}9.80 \pm 4.17 \\
\quad(395)\end{array}$ & $\begin{array}{l}10.51 \pm 7.76 \\
\quad(383)\end{array}$ & 0.115 & $\begin{array}{l}10.26 \pm 8.16 \\
(220)\end{array}$ & 0.362 & $\begin{array}{l}10.77 \pm 7.24 \\
\quad(159)\end{array}$ & 0.050 \\
\hline Folate: ng/mL (n) & $\begin{array}{l}8.85 \pm 8.06 \\
\quad(392)\end{array}$ & $\begin{array}{l}7.77 \pm 6.65 \\
\quad(381)\end{array}$ & 0.043 & $\begin{array}{l}8.06 \pm 7.19 \\
(220)\end{array}$ & 0.226 & $\begin{array}{l}7.35 \pm 5.85 \\
\quad(157)\end{array}$ & 0.034 \\
\hline \multicolumn{8}{|l|}{ Tumor size: $n(\%)$} \\
\hline$<5 \mathrm{~cm}$ & - & $181(40.2)$ & NA & $92(35.4)$ & NA & $87(46.8)$ & NA \\
\hline$\geq 5 \mathrm{~cm}$ & - & $269(59.8)$ & NA & $168(64.6)$ & NA & $99(53.2)$ & NA \\
\hline \multicolumn{8}{|l|}{ TNM stage: $n(\%)$} \\
\hline I & - & $42(9.3)$ & NA & $22(8.5)$ & NA & $20(10.8)$ & NA \\
\hline II & - & $189(42.0)$ & NA & $115(44.2)$ & NA & $71(38.2)$ & NA \\
\hline III & - & $173(38.4)$ & NA & $94(36.2)$ & NA & 79 (42.5) & NA \\
\hline IV & - & $46(10.2)$ & NA & 29 (11.2) & NA & $16(8.6)$ & NA \\
\hline
\end{tabular}

Abbreviations: CRC, colorectal cancer; SD, standard deviation; BMI, body mass index; HDL-C, high density lipoprotein-cholesterol; MTHFR, methylenetetrahydrofolate reductase; TNM, tumor node metastasis. $p$-values were calculated by chi-square test for categorical data and two-sided $t$-test for continuous data. NA: Statistics cannot be processed because there is no comparison group.

\subsection{Genotype Frequencies of TS 3'-UTR Variants}

Table 2 shows the distributions of genotypes for TS 3'-UTR polymorphisms in CRC patients and control subjects. The genotype frequencies in both groups were consistent with expectations under Hardy-Weinberg equilibrium (HWE). In the total CRC group, TS $1170 \mathrm{AG}(\mathrm{AOR}=1.55,95 \% \mathrm{CI}=1.13-2.11)$ and $1170 \mathrm{GG}(\mathrm{AOR}=3.19,95 \% \mathrm{CI}=1.91-5.34)$ were found to be significantly associated with CRC susceptibility. We then divided CRC patients into two subgroups, consisting of those with colon and rectal cancer. For the colon subgroup, $\mathrm{TS} 1170 \mathrm{AG}(\mathrm{AOR}=1.73,95 \% \mathrm{CI}=1.20-2.50)$ and $1170 \mathrm{GG}(\mathrm{AOR}=4.31,95 \%$ $\mathrm{CI}=2.42-7.66)$ were significantly associated with CRC susceptibility, and in the rectum subgroup, 1170GG (AOR $=2.07,95 \% \mathrm{CI}=2.42-7.66)$ was significantly associated with CRC susceptibility. No statistically significant associations were found for TS1100T $>C$ genotypes.

\subsection{Effects of Combined Genotypes and Allelic Gene-Gene Interactions for TS 3'-UTR Variants on CRC Incident Rates}

To evaluate possible combined effects between TS 3'-UTR polymorphic loci on CRC incidence, we performed logistic regression on the combined genotypes (Table 3). In the total CRC group, TS 1100TT-1170GG (AOR $=5.24 ; 95 \% \mathrm{CI}=2.67-10.27), 1100 \mathrm{TC}-1170 \mathrm{AG}$ $(\mathrm{AOR}=3.06 ; 95 \% \mathrm{CI}=1.73-5.43)$, and 1100CC-1170AA $(\mathrm{AOR}=3.00 ; 95 \% \mathrm{CI}=1.45-6.21)$ showed significant AOR values $>3.00$. In the colon and rectum subgroups, TS 1100TT1170GG $(\mathrm{AOR}=6.10 ; 95 \% \mathrm{CI}=2.81-13.23, \mathrm{AOR}=4.04 ; 95 \% \mathrm{CI}=1.69-9.69$, respectively $)$, 1100TC-1170AG $(\mathrm{AOR}=3.09 ; 95 \% \mathrm{CI}=1.59-6.00, \mathrm{AOR}=3.43 ; 95 \% \mathrm{CI}=1.57-7.45$, respectively), and 1100CC-1170AA ( $\mathrm{AOR}=3.12 ; 95 \% \mathrm{CI}=1.34-7.28, \mathrm{AOR}=2.98 ; 95 \% \mathrm{CI}=1.11-8.01$, respectively) showed significant $\mathrm{AOR}$ values $>3.00$. For the TS haplotypes, in the total CRC group, TS 1100T-1170G (AOR $=2.00 ; 95 \% \mathrm{CI}=1.56-2.55)$ and $1100 \mathrm{C}-1170 \mathrm{~A}$ (AOR $=1.51 ; 95 \% \mathrm{CI}=1.17-1.96)$ were associated with increased CRC incidence rates. In the colon and rectum subgroups, TS 1100T-1170G $(\mathrm{AOR}=2.25 ; 95 \% \mathrm{CI}=1.69-3.00, \mathrm{AOR}=1.71$; $95 \% \mathrm{CI}=1.24-2.34$, respectively $)$ and TS 1100C-1170A ( $\mathrm{AOR}=1.57 ; 95 \% \mathrm{CI}=1.16-2.13$, $\mathrm{AOR}=1.49 ; 95 \% \mathrm{CI}=1.08-2.07$, respectively) were significantly associated with CRC susceptibility. 
Table 2. Genotype frequencies of TS 3'-UTR polymorphisms between CRC patients and control subjects.

\begin{tabular}{|c|c|c|c|c|c|c|c|c|c|c|}
\hline Genotypes & $\begin{array}{c}\text { Control } \\
(n=400)\end{array}$ & $\begin{array}{c}\text { CRC } \\
(n=450)\end{array}$ & $\begin{array}{c}\text { AOR }(95 \% \\
\text { CI) }\end{array}$ & $p$ & $\begin{array}{l}\text { Colon } \\
(n=260)\end{array}$ & $\begin{array}{c}\text { AOR } \\
(95 \% \text { CI })\end{array}$ & $p$ & $\begin{array}{l}\text { Rectum } \\
(n=186)\end{array}$ & $\begin{array}{c}\text { AOR } \\
(\mathbf{9 5 \%} \mathrm{CI})\end{array}$ & $p$ \\
\hline & & & & & TS $1100 \mathrm{~T}>\mathrm{C}$ & & & & & \\
\hline $\mathrm{TT}$ & $195(48.8)$ & $223(49.6)$ & 1.00 (ref) & NA & $134(51.5)$ & 1.00 (ref) & NA & $86(46.2)$ & 1.00 (ref) & NA \\
\hline TC & $177(44.3)$ & $189(42.0)$ & $\begin{array}{c}0.99 \\
(0.73-1.33)\end{array}$ & 0.922 & $105(40.4)$ & $\begin{array}{c}0.88 \\
(0.62-1.25)\end{array}$ & 0.482 & $84(45.2)$ & $\begin{array}{c}1.21 \\
(0.82-1.78)\end{array}$ & 0.345 \\
\hline $\mathrm{CC}$ & $28(7.0)$ & $38(8.4)$ & $\begin{array}{c}1.27 \\
(0.73-2.22)\end{array}$ & 0.401 & $21(8.1)$ & $\begin{array}{c}1.25 \\
(0.66-2.37)\end{array}$ & 0.489 & $16(8.6)$ & $\begin{array}{c}1.28 \\
(0.62-2.64)\end{array}$ & 0.511 \\
\hline AA & $195(48.8)$ & 155 (34.4) & 1.00 (ref) & NA & $\begin{array}{c}\text { TS } 1170 A>G \\
82(31.5)\end{array}$ & 1.00 (ref) & NA & $71(38.2)$ & 1.00 (ref) & NA \\
\hline AG & $176(44.0)$ & $224(49.8)$ & $\begin{array}{c}1.55 \\
(1.13-2.11)\end{array}$ & 0.006 & $132(50.8)$ & $\begin{array}{c}1.73 \\
(1.20-2.50)\end{array}$ & 0.003 & 90 (48.4) & $\begin{array}{c}1.42 \\
(0.95-2.11)\end{array}$ & 0.085 \\
\hline GG & $29(7.3)$ & $71(15.8)$ & $\begin{array}{c}3.19 \\
(1.91-5.34)\end{array}$ & $<0.001$ & 46 (17.7) & $\begin{array}{c}4.31 \\
(2.42-7.66)\end{array}$ & $<0.001$ & $25(13.4)$ & $\begin{array}{c}2.07 \\
(1.08-3.95)\end{array}$ & 0.028 \\
\hline
\end{tabular}

Abbreviations: MTHFR, methylenetetrahydrofolate reductase; TS, thymidylate synthase; CRC, colorectal cancer; AOR, adjusted odds ratio (adjusted by age, gender, hypertension, diabetes mellitus, body mass index, and high density lipoprotein-cholesterol); $\mathrm{CI}$, confidence interval. NA: Statistical processing was not possible because the corresponding value was a reference.

Table 3. Combined genotype and haplotype frequencies of TS $3^{\prime}-\mathrm{UTR}$ polymorphisms between CRC patients and control subjects.

\begin{tabular}{|c|c|c|c|c|c|c|c|c|c|c|}
\hline Genotypes & $\begin{array}{c}\text { Control } \\
(n=400)\end{array}$ & $\begin{array}{c}\text { CRC } \\
(n=450)\end{array}$ & $\begin{array}{c}\text { AOR } \\
(95 \% \text { CI })\end{array}$ & $p$ & $\begin{array}{c}\text { Colon } \\
(n=260)\end{array}$ & $\begin{array}{c}\text { AOR } \\
(95 \% \mathrm{CI})\end{array}$ & $p$ & $\begin{array}{l}\text { Rectum } \\
(n=186)\end{array}$ & $\begin{array}{c}\text { AOR } \\
(95 \% \mathrm{CI})\end{array}$ & $p$ \\
\hline \multicolumn{11}{|c|}{ TS Genotype } \\
\hline $\begin{array}{l}\text { 1100TT- } \\
\text { 1170AA }\end{array}$ & $63(15.8)$ & $39(8.7)$ & 1.00 (ref) & NA & $23(8.8)$ & 1.00 (ref) & NA & $15(8.1)$ & 1.00 (ref) & NA \\
\hline $\begin{array}{l}\text { 1100TT- } \\
1170 \mathrm{AG}\end{array}$ & $103(25.8)$ & $113(25.1)$ & $\begin{array}{c}1.81 \\
(1.07-3.06)\end{array}$ & 0.027 & $65(25.0)$ & $\begin{array}{c}1.75 \\
(0.95-3.25)\end{array}$ & 0.074 & $46(24.7)$ & $\begin{array}{c}1.94 \\
(0.96-3.93)\end{array}$ & 0.067 \\
\hline $\begin{array}{l}\text { 1100TT- } \\
\text { 1170GG }\end{array}$ & $29(7.3)$ & $71(15.8)$ & $\begin{array}{c}5.24 \\
(2.67-10.27)\end{array}$ & $<0.001$ & $46(17.7)$ & $\begin{array}{c}6.10 \\
(2.81-13.23)\end{array}$ & $<0.001$ & 25 (13.4) & $\begin{array}{c}4.04 \\
(1.69-9.69)\end{array}$ & 0.002 \\
\hline $\begin{array}{l}1100 \mathrm{TC}- \\
1170 \mathrm{AA}\end{array}$ & $104(26.0)$ & $78(17.3)$ & $\begin{array}{c}1.40 \\
(0.82-2.40)\end{array}$ & 0.220 & 38 (14.6) & $\begin{array}{c}1.10 \\
(0.57-2.12)\end{array}$ & 0.785 & $40(21.5)$ & $\begin{array}{c}1.85 \\
(0.90-3.79)\end{array}$ & 0.095 \\
\hline $\begin{array}{l}1100 \mathrm{TC}- \\
1170 \mathrm{AG}\end{array}$ & 73 (18.3) & $111(24.7)$ & $\begin{array}{c}3.06 \\
(1.73-5.43)\end{array}$ & $<0.001$ & $67(25.8)$ & $\begin{array}{c}3.09 \\
(1.59-6.00)\end{array}$ & $<0.001$ & $44(23.7)$ & $\begin{array}{c}3.43 \\
(1.57-7.45)\end{array}$ & 0.002 \\
\hline $\begin{array}{l}\text { 1100CC- } \\
\text { 1170AA }\end{array}$ & $28(7.0)$ & $38(8.4)$ & $\begin{array}{c}3.00 \\
(1.45-6.21)\end{array}$ & 0.003 & $21(8.1)$ & $\begin{array}{c}3.12 \\
(1.34-7.28)\end{array}$ & 0.009 & $16(8.6)$ & $\begin{array}{c}2.98 \\
(1.11-8.01)\end{array}$ & 0.030 \\
\hline \multicolumn{11}{|c|}{ TS haplotype } \\
\hline $\begin{array}{l}1100 \mathrm{~T}- \\
1170 \mathrm{~A}\end{array}$ & $333(41.6)$ & $269(29.9)$ & 1.00 (ref) & NA & $149(28.7)$ & 1.00 (ref) & NA & $116(31.2)$ & 1.00 (ref) & NA \\
\hline $\begin{array}{l}1100 \mathrm{~T}- \\
1170 \mathrm{G}\end{array}$ & $234(29.3)$ & $366(40.7)$ & $\begin{array}{c}2.00 \\
(1.56-2.55)\end{array}$ & $<0.001$ & $224(43.1)$ & $\begin{array}{c}2.25 \\
(1.69-3.00)\end{array}$ & $<0.001$ & $140(37.6)$ & $\begin{array}{c}1.71 \\
(1.24-2.34)\end{array}$ & $<0.001$ \\
\hline $\begin{array}{l}1100 \mathrm{C}- \\
1170 \mathrm{~A}\end{array}$ & $233(29.1)$ & $265(29.4)$ & $\begin{array}{c}1.51 \\
(1.17-1.96)\end{array}$ & 0.002 & $147(28.3)$ & $\begin{array}{c}1.57 \\
(1.16-2.13)\end{array}$ & 0.004 & $116(31.2)$ & $\begin{array}{c}1.49 \\
(1.08-2.07)\end{array}$ & 0.017 \\
\hline
\end{tabular}

Adjusted by age, gender, hypertension, diabetes mellitus, body mass index, and high density lipoprotein-cholesterol. NA: Statistical processing was not possible because the corresponding value was a reference.

\subsection{Stratified Effects of TS 3'-UTR Polymorphisms on CRC Incidence}

To determine whether certain alleles were associated with CRC incidence in specific subsets of patients, we conducted a stratified analysis of the data according to age, gender, hypertension (HTN), diabetes mellitus (DM), tumor node metastasis (TNM) stage, body mass index (BMI), high density lipoprotein-cholesterol (HDL-C), homocysteine (Hcy), and folate (FA) (Table 4). To establish cut-off values in the ranges of Hcy and FA serum levels, we selected upper and lower $25 \%$ cut-offs for Hcy and FA, respectively. These values correspond to $11.7 \mu \mathrm{mol} / \mathrm{L}$ for Hcy and $4.58 \mathrm{ng} / \mathrm{mL}$ for FA. Tables 4 and 5 contain results from our stratified analysis of constructed TS haplotypes and TS combined genotypes. For the TS 1100/1170 combined genotypes, common subset-specific associations for 1100TC-1170AG, and 1100CC-1170AA were found for the male, TNM I or II stage, HTN, Hcy $<11.7 \mu \mathrm{mol} / \mathrm{L}$ and FA $\geq 4.58 \mathrm{ng} / \mathrm{mL}$ subgroups; specific associations for TS 1100TT-1170GG were found for the female, TNM I or II, BMI $\geq 25 \mathrm{~kg} / \mathrm{m}^{2}, \mathrm{Hcy}<11.7 \mu \mathrm{mol} / \mathrm{L}$, and FA $\geq 4.58 \mathrm{ng} / \mathrm{mL}$ subgroups; and specific associations for TS 1100TT-1170AG were found for the tumor size $<5 \mathrm{~cm}$, TNM I or II BMI $<25 \mathrm{~kg} / \mathrm{m}^{2}, \mathrm{HDL}-\mathrm{C} \geq 40(\mathrm{M}) / 50$ (F) mg/dL, Hcy $<11.7 \mu \mathrm{mol} / \mathrm{L}$, 
and FA $\geq 4.58 \mathrm{ng} / \mathrm{mL}$ subgroups (Table 4). TS 1100C-1170A haplotypes displayed common subset-specific associations with the age $\geq 62$ years, male, $\geq 5$-cm tumor, TNM I or II stage, HTN, DM, BMI $\left(<25 \mathrm{~kg} / \mathrm{m}^{2}\right)$, HDL-C $\geq 40$ (male) $/ 50$ (female) $\mathrm{mg} / \mathrm{dL}, \mathrm{Hcy} \geq 11.7 \mu \mathrm{mol} / \mathrm{L}$ and FA ( $\geq 4.58 \mathrm{ng} / \mathrm{mL})$ subgroups (Table 5 ).

\subsection{Gene-Environment Combined Effects of TS 3'-UTR Polymorphisms on CRC Incidence}

Because cancer risk and prognosis are determined by a complex interplay of genetic and environmental factors, we calculated the combined gene-environment effects on CRC incidence (Supplemental Table S1). To perform this gene-environment interaction analysis for CRC incidence rates, we selected the environmental factors, such as HTN, DM, HDLC, Hcy, and FA, which showed statistically significant differences in subjects with total $\mathrm{CRC}$, colon, or rectal cancer relative to controls. Of the single genotypes, TS 1170AG+GG displayed strong gene-environment combined effects with $\mathrm{HTN}$ and FA $<4.58 \mathrm{ng} / \mathrm{mL}$ on CRC incidence. For the TS 1100/1170 combined genotypes, TS 1100TT-1170GG, 1100TC$1170 \mathrm{AG}$, and 1100CC-1170AA showed strong gene-environment combined effects with HTN, DM, and HDL-C < 40 (male)/50 (female) $\mathrm{mg} / \mathrm{dL}$ on CRC prevalence.

Table 4. Analysis of CRC incidence and association in specific patient subsets.

\begin{tabular}{|c|c|c|c|c|c|c|c|c|c|c|}
\hline \multirow[b]{2}{*}{ Subsets } & \multicolumn{2}{|c|}{ TS 1100TT-1170AG } & \multicolumn{2}{|c|}{ TS 1100TT-1170GG } & \multicolumn{2}{|c|}{ TS 1100TC-1170AA } & \multicolumn{2}{|c|}{ TS 1100TC-1170AG } & \multicolumn{2}{|c|}{ TS 1100CC-1170AA } \\
\hline & $\begin{array}{c}\text { AOR } \\
(95 \% \text { CI })\end{array}$ & $p$ & $\begin{array}{c}\text { AOR } \\
(95 \% \text { CI })\end{array}$ & $p$ & $\begin{array}{c}\text { AOR } \\
(95 \% \text { CI })\end{array}$ & $p$ & $\begin{array}{c}\text { AOR } \\
(95 \% \text { CI })\end{array}$ & $p$ & $\begin{array}{c}\text { AOR } \\
(95 \% \text { CI })\end{array}$ & $p$ \\
\hline Age $<62$ years & $\begin{array}{c}2.08 \\
(0.98-4.43)\end{array}$ & 0.058 & $\begin{array}{c}3.38 \\
(1.34-8.52)\end{array}$ & 0.010 & $\begin{array}{c}1.07 \\
(0.50-2.32)\end{array}$ & 0.856 & $\begin{array}{c}2.78 \\
(1.24-6.24)\end{array}$ & 0.013 & $\begin{array}{c}1.48 \\
(0.53-4.14)\end{array}$ & 0.454 \\
\hline$\geq 62$ years & $\begin{array}{c}1.79 \\
(0.85-3.77)\end{array}$ & 0.126 & $\begin{array}{c}7.62 \\
(2.68-21.68)\end{array}$ & $<0.001$ & $\begin{array}{c}1.87 \\
(0.85-4.12)\end{array}$ & 0.122 & $\begin{array}{c}3.08 \\
(1.35-7.04)\end{array}$ & 0.008 & $\begin{array}{c}6.63 \\
(2.10-20.97)\end{array}$ & 0.001 \\
\hline Gender Male & $\begin{array}{c}2.12 \\
(0.90-5.01)\end{array}$ & 0.086 & $\begin{array}{c}4.61 \\
(1.80-11.78)\end{array}$ & 0.001 & $\begin{array}{c}1.94 \\
(0.80-4.67)\end{array}$ & 0.142 & $\begin{array}{c}3.57 \\
(1.51-8.43)\end{array}$ & 0.004 & $\begin{array}{c}3.45 \\
(1.16-10.29)\end{array}$ & 0.026 \\
\hline Female & $\begin{array}{c}1.62 \\
(0.82-3.18)\end{array}$ & 0.162 & $\begin{array}{c}6.23 \\
(2.24-17.34)\end{array}$ & $<0.001$ & $\begin{array}{c}1.02 \\
(0.50-2.07)\end{array}$ & 0.953 & $\begin{array}{c}2.70 \\
(1.21-6.04)\end{array}$ & 0.015 & $\begin{array}{c}2.91 \\
(1.06-8.01)\end{array}$ & 0.039 \\
\hline $\mathrm{TS}<5 \mathrm{~cm}$ & $\begin{array}{c}2.13 \\
(1.06-4.27)\end{array}$ & 0.034 & $\begin{array}{c}5.83 \\
(2.37-14.32)\end{array}$ & $<0.001$ & $\begin{array}{c}1.71 \\
(0.82-3.57)\end{array}$ & 0.154 & $\begin{array}{c}3.88 \\
(1.74-8.65)\end{array}$ & $<0.001$ & $\begin{array}{c}2.41 \\
(0.83-7.00)\end{array}$ & 0.106 \\
\hline$\geq 5 \mathrm{~cm}$ & $\begin{array}{c}1.65 \\
(0.88-3.09)\end{array}$ & 0.120 & $\begin{array}{c}5.03 \\
(2.35-10.80)\end{array}$ & $<0.001$ & $\begin{array}{c}1.24 \\
(0.65-2.37)\end{array}$ & 0.506 & $\begin{array}{c}2.97 \\
(1.53-5.77)\end{array}$ & 0.001 & $\begin{array}{c}3.71 \\
(1.61-8.54)\end{array}$ & 0.002 \\
\hline TNM I + II & $\begin{array}{c}2.20 \\
(1.13-4.31)\end{array}$ & 0.021 & $\begin{array}{c}6.24 \\
(2.74-14.18)\end{array}$ & $<0.001$ & $\begin{array}{c}1.63 \\
(0.81-3.25)\end{array}$ & 0.169 & $\begin{array}{c}3.87 \\
(1.89-7.90)\end{array}$ & $<0.001$ & $\begin{array}{c}3.35 \\
(1.33-8.43)\end{array}$ & 0.010 \\
\hline III + IV & $\begin{array}{c}1.53 \\
(0.81-2.89)\end{array}$ & 0.194 & $\begin{array}{c}4.56 \\
(2.01-10.36)\end{array}$ & $<0.001$ & $\begin{array}{c}1.22 \\
(0.62-2.37)\end{array}$ & 0.564 & $\begin{array}{c}2.62 \\
(1.28-5.34)\end{array}$ & 0.008 & $\begin{array}{c}3.00 \\
(1.23-7.29)\end{array}$ & 0.015 \\
\hline HTN No & $\begin{array}{c}1.40 \\
(0.66-3.00)\end{array}$ & 0.379 & $\begin{array}{c}4.88 \\
(1.87-12.78)\end{array}$ & 0.001 & $\begin{array}{c}1.13 \\
(0.53-2.44)\end{array}$ & 0.752 & $\begin{array}{c}2.60 \\
(1.17-5.79)\end{array}$ & 0.019 & $\begin{array}{c}2.07 \\
(0.73-5.85)\end{array}$ & 0.172 \\
\hline Yes & $\begin{array}{c}2.43 \\
(1.16-5.11)\end{array}$ & 0.019 & $\begin{array}{c}5.61 \\
(2.10-14.96)\end{array}$ & $<0.001$ & $\begin{array}{c}2.00 \\
(0.91-4.36)\end{array}$ & 0.083 & $\begin{array}{c}3.86 \\
(1.64-9.07)\end{array}$ & 0.002 & $\begin{array}{c}4.59 \\
(1.58-13.31)\end{array}$ & 0.005 \\
\hline DM No & $\begin{array}{c}3.29 \\
(1.38-7.84)\end{array}$ & 0.007 & $\begin{array}{c}5.93 \\
(2.27-15.50)\end{array}$ & $<0.001$ & $\begin{array}{c}2.44 \\
(0.96-6.20)\end{array}$ & 0.061 & $\begin{array}{c}3.56 \\
(1.43-8.86)\end{array}$ & 0.006 & $\begin{array}{c}2.09 \\
(0.74-5.95)\end{array}$ & 0.167 \\
\hline Yes & $\begin{array}{c}1.11 \\
(0.55-2.23)\end{array}$ & 0.773 & $\begin{array}{c}4.60 \\
(1.70-12.43)\end{array}$ & 0.003 & $\begin{array}{c}0.93 \\
(0.45-1.92)\end{array}$ & 0.840 & $\begin{array}{c}2.62 \\
(1.22-5.65)\end{array}$ & 0.014 & $\begin{array}{c}3.85 \\
(1.28-11.54)\end{array}$ & 0.016 \\
\hline $\mathrm{BMI}<25 \mathrm{~kg} / \mathrm{m}^{2}$ & $\begin{array}{c}2.13 \\
(1.13-3.99)\end{array}$ & 0.019 & $\begin{array}{c}4.96 \\
(2.27-10.84)\end{array}$ & $<0.001$ & $\begin{array}{c}1.27 \\
(0.67-2.41)\end{array}$ & 0.470 & $\begin{array}{c}2.67 \\
(1.39-5.12)\end{array}$ & 0.003 & $\begin{array}{c}3.96 \\
(1.67-9.39)\end{array}$ & 0.002 \\
\hline$\geq 25 \mathrm{~kg} / \mathrm{m}^{2}$ & $\begin{array}{c}1.26 \\
(0.48-3.33)\end{array}$ & 0.643 & $\begin{array}{c}6.41 \\
(1.41-29.18)\end{array}$ & 0.016 & $\begin{array}{c}1.85 \\
(0.66-5.17)\end{array}$ & 0.240 & $\begin{array}{c}5.07 \\
(1.41-18.22)\end{array}$ & 0.013 & $\begin{array}{c}1.57 \\
(0.35-7.08)\end{array}$ & 0.558 \\
\hline HDL-C $<40(\mathrm{M}) / 50(\mathrm{~F})$ & $\begin{array}{c}1.37 \\
(0.57-3.26)\end{array}$ & 0.479 & $\begin{array}{c}2.82 \\
(0.90-8.89)\end{array}$ & 0.077 & $\begin{array}{c}0.96 \\
(0.38-2.45)\end{array}$ & 0.934 & $\begin{array}{c}3.71 \\
(1.33-10.39)\end{array}$ & 0.013 & $\begin{array}{c}1.44 \\
(0.35-5.98)\end{array}$ & 0.618 \\
\hline$\geq 40(\mathrm{M}) / 50(\mathrm{~F})$ & $\begin{array}{c}2.12 \\
(1.07-4.20)\end{array}$ & 0.031 & $\begin{array}{c}7.69 \\
(3.21-18.44)\end{array}$ & $<0.001$ & $\begin{array}{c}1.64 \\
(0.82-3.29)\end{array}$ & 0.163 & $\begin{array}{c}3.02 \\
(1.48-6.16)\end{array}$ & 0.002 & $\begin{array}{c}3.60 \\
(1.50-8.66)\end{array}$ & 0.004 \\
\hline Hcy $<11.7 \mu \mathrm{mol} / \mathrm{L}$ & $\begin{array}{c}2.11 \\
(1.10-4.07)\end{array}$ & 0.025 & $\begin{array}{c}5.45 \\
(2.40-12.35)\end{array}$ & $<0.001$ & $\begin{array}{c}1.41 \\
(0.73-2.72)\end{array}$ & 0.308 & $\begin{array}{c}2.88 \\
(1.40-5.96)\end{array}$ & 0.004 & $\begin{array}{c}3.14 \\
(1.25-7.89)\end{array}$ & 0.015 \\
\hline$\geq 11.7 \mu \mathrm{mol} / \mathrm{L}$ & $\begin{array}{c}0.97 \\
(0.29-3.20)\end{array}$ & 0.957 & $\begin{array}{c}4.14 \\
(0.96-17.85)\end{array}$ & 0.057 & $\begin{array}{c}1.87 \\
(0.53-6.55)\end{array}$ & 0.327 & $\begin{array}{c}2.88 \\
(0.82-10.14)\end{array}$ & 0.100 & $\begin{array}{c}4.12 \\
(0.73-23.18)\end{array}$ & 0.108 \\
\hline $\mathrm{FA}<4.58 \mathrm{ng} / \mathrm{mL}$ & $\begin{array}{c}1.52 \\
(0.47-4.86)\end{array}$ & 0.485 & $\begin{array}{c}3.31 \\
(0.59-18.52)\end{array}$ & 0.173 & $\begin{array}{c}0.92 \\
(0.27-3.08)\end{array}$ & 0.888 & $\begin{array}{c}2.44 \\
(0.71-8.31)\end{array}$ & 0.156 & $\begin{array}{c}3.47 \\
(0.55-21.80)\end{array}$ & 0.184 \\
\hline$\geq 4.58 \mathrm{ng} / \mathrm{mL}$ & $\begin{array}{c}2.00 \\
(1.02-3.92)\end{array}$ & 0.043 & $\begin{array}{c}8.36 \\
(3.48-20.08)\end{array}$ & $<0.001$ & $\begin{array}{c}1.53 \\
(0.77-3.04)\end{array}$ & 0.228 & $\begin{array}{c}3.43 \\
(1.62-7.27)\end{array}$ & 0.001 & $\begin{array}{c}3.84 \\
(1.43-10.35)\end{array}$ & 0.008 \\
\hline
\end{tabular}

TS, Tumor size; TNM, Tumor Node Metastasis stage; HTN, hypertension; DM, diabetes mellitus; BMI, body mass index; HDL-C, high density lipoprotein-cholesterol; M, male; F, female; Hcy, homocysteine; FA, folate.11.7 was upper $25 \%$ cut-off value of Hcy in total participants. 4.58 was lower $25 \%$ cut-off value of FA in total participants. Adjusted by age, gender, HTN, DM, BMI and HDL-C. 
Table 5. Analysis of CRC incidence and association in haplotypes of TS 1100/1170.

\begin{tabular}{|c|c|c|c|c|}
\hline \multirow[b]{2}{*}{ Subsets } & \multicolumn{2}{|c|}{ 1100T-1170G } & \multicolumn{2}{|c|}{ 1100C-1170A } \\
\hline & AOR $(95 \% \mathrm{CI})$ & $p$ & AOR $(95 \%$ CI) & $p$ \\
\hline Age $<62$ years & $1.91(1.35-2.72)$ & $<0.001$ & $1.22(0.85-1.76)$ & 0.274 \\
\hline$\geq 62$ years & $1.95(1.37-2.79)$ & $<0.001$ & $1.75(1.20-2.53)$ & 0.003 \\
\hline Gender Male & $2.08(1.43-3.02)$ & $<0.001$ & $1.72(1.16-2.56)$ & 0.007 \\
\hline Female & $1.91(1.37-2.68)$ & $<0.001$ & $1.37(0.97-1.93)$ & 0.074 \\
\hline Tumor size $<5 \mathrm{~cm}$ & $1.86(1.36-2.53)$ & $<0.001$ & $1.32(0.95-1.84)$ & 0.103 \\
\hline$\geq 5 \mathrm{~cm}$ & $2.10(1.57-2.81)$ & $<0.001$ & $1.70(1.26-2.30)$ & $<0.001$ \\
\hline TNM stage I + II & $2.15(1.60-2.90)$ & $<0.001$ & $1.57(1.15-2.15)$ & 0.005 \\
\hline III + IV & $1.82(1.35-2.46)$ & $<0.001$ & $1.47(1.07-2.02)$ & 0.017 \\
\hline HTN No & $1.99(1.40-2.83)$ & $<0.001$ & $1.43(0.99-2.07)$ & 0.059 \\
\hline Yes & $1.97(1.39-2.80)$ & $<0.001$ & $1.64(1.14-2.35)$ & 0.008 \\
\hline DM No & $2.04(1.44-2.90)$ & $<0.001$ & $1.27(0.87-1.84)$ & 0.214 \\
\hline Yes & $1.90(1.34-2.70)$ & $<0.001$ & $1.77(1.23-2.54)$ & 0.002 \\
\hline $\mathrm{BMI}<25 \mathrm{~kg} / \mathrm{m}^{2}$ & $2.00(1.51-2.67)$ & $<0.001$ & $1.48(1.10-1.99)$ & 0.009 \\
\hline$\geq 25 \mathrm{~kg} / \mathrm{m}^{2}$ & $1.97(1.20-3.23)$ & 0.008 & $1.66(0.98-2.82)$ & 0.061 \\
\hline $\mathrm{HDL}-\mathrm{C}<\overline{40}(\mathrm{M}) / 50(\mathrm{~F}) \mathrm{mg} / \mathrm{dL}$ & $1.84(1.17-2.89)$ & 0.008 & $1.35(0.83-2.19)$ & 0.221 \\
\hline$\geq 40(\mathrm{M}) / 50(\mathrm{~F}) \mathrm{mg} / \mathrm{dL}$ & $2.09(1.55-2.81)$ & $<0.001$ & $1.56(1.15-2.12)$ & 0.005 \\
\hline Hcy $<11.7 \mu \mathrm{mol} / \mathrm{L}$ & $1.97(1.46-2.66)$ & $<0.001$ & $1.35(0.99-1.83)$ & 0.060 \\
\hline$\geq 11.7 \mu \mathrm{mol} / \mathrm{L}$ & $1.80(1.05-3.08)$ & 0.031 & $1.92(1.08-3.43)$ & 0.027 \\
\hline $\mathrm{FA}<4.58 \mathrm{ng} / \mathrm{mL}$ & $1.75(1.00-3.07)$ & 0.049 & $1.52(0.87-2.65)$ & 0.141 \\
\hline$\geq 4.58 \mathrm{ng} / \mathrm{mL}$ & $2.12(1.57-2.86)$ & $<0.001$ & $1.50(1.09-2.06)$ & 0.013 \\
\hline
\end{tabular}

HTN, hypertension; DM, diabetes mellitus; BMI, body mass index; HDL-C, high density lipoprotein-cholesterol; M, male; F, female; Hcy, homocysteine; FA, folate. The upper $25 \%$ cut-off value of Hcy in total participants was 11.7 . The lower $25 \%$ cut-off value of FA in total participants was 4.58

\subsection{CRC Progression According to TS 3'-UTR Polymorphisms}

To identify genetic associations with CRC prognosis, we conducted a survival analysis for OS and RFS according to TS 3'-UTR polymorphisms. From our total cohort, 100 CRC patients had an insufficient medical history and were therefore excluded from this analysis. Characteristics of the 350 subjects included in this survival analysis are summarized in Supplemental Tables S2 and S3 which show the OS and RFS rates for various TS 3'UTR polymorphisms. From this analysis, we found a significant association between TS $1100 \mathrm{TC}+\mathrm{CC}$ and poor OS (AHR $=1.82 ; 95 \% \mathrm{CI}=1.07-3.78)$ and $\mathrm{RFS}$ rates $(\mathrm{AHR}=2.00 ; 95 \%$ $\mathrm{CI}=1.19-3.35$ ) (Figure 1). Supplemental Table S4 presents AHR values of prognosis risk factors according to TS 3'-UTR polymorphisms. TNM stage, tumor differentiation, HDL-C, and FA significantly affected OS and RFS rate of CRC patients. The poorest AHR values for OS and RFS by HDL-C levels occurred based on the TS 1100TC-1170AG combined genotype. Of AHR values for OS and RFS by plasma FA levels, the TS 1100TC-1170AA combined genotype showed significantly higher values than others.

\subsection{Expression of TS 3'-UTR Polymorphisms}

We quantified expression of TS in tissue samples and determined whether we could detect differences in expression based on the TS 3 '-UTR polymorphisms and tested haplotypes (Figure 2, Supplemental Table S5). Compared with the wild-type (WT) 1100TT genotype, expression of $1100 \mathrm{TC}$ and $1100 \mathrm{CC}$ was found to be significantly increased in tumor tissue. In the case of TS $1170 \mathrm{~A}>\mathrm{G}$, we detected increased expression of $1170 \mathrm{AG}$ and 1170GG relative to 1170AA (WT) in tumors, similar to what we observed for the TS $1100 \mathrm{~T}>\mathrm{C}$ genotype. In addition, we found that the TS 1100T-1170G and 1100C-1170A haplotypes were associated with increased TS expression. Thus, our data indicate that expression of the mutant type was significantly increased in CRC for both TS 1100 and TS 1170. 

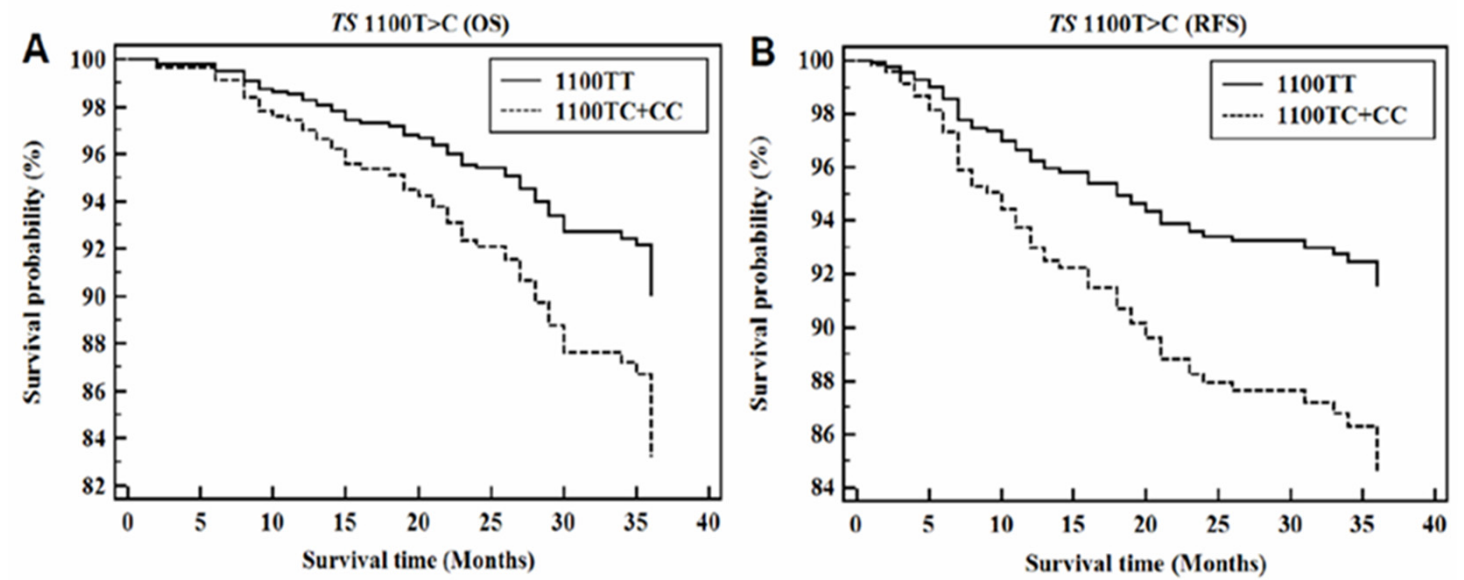

Figure 1. Cox proportional-hazard regression analysis for TS $1100 \mathrm{~T}>\mathrm{C}$. (A) Overall survival (OS) curve for TS $1100 \mathrm{~T}>\mathrm{C}$ (Dominant model; $\mathrm{AHR}=1.82 ; 95 \% \mathrm{CI}=1.07-3.08 ; p=0.027$ ). (B) Relapse-free survival (RFS) curve for TS $1100 \mathrm{~T}>\mathrm{C}$ (Dominant model; AHR $=2.00 ; 95 \% \mathrm{CI}=1.19-3.35 ; p=0.009$ ).

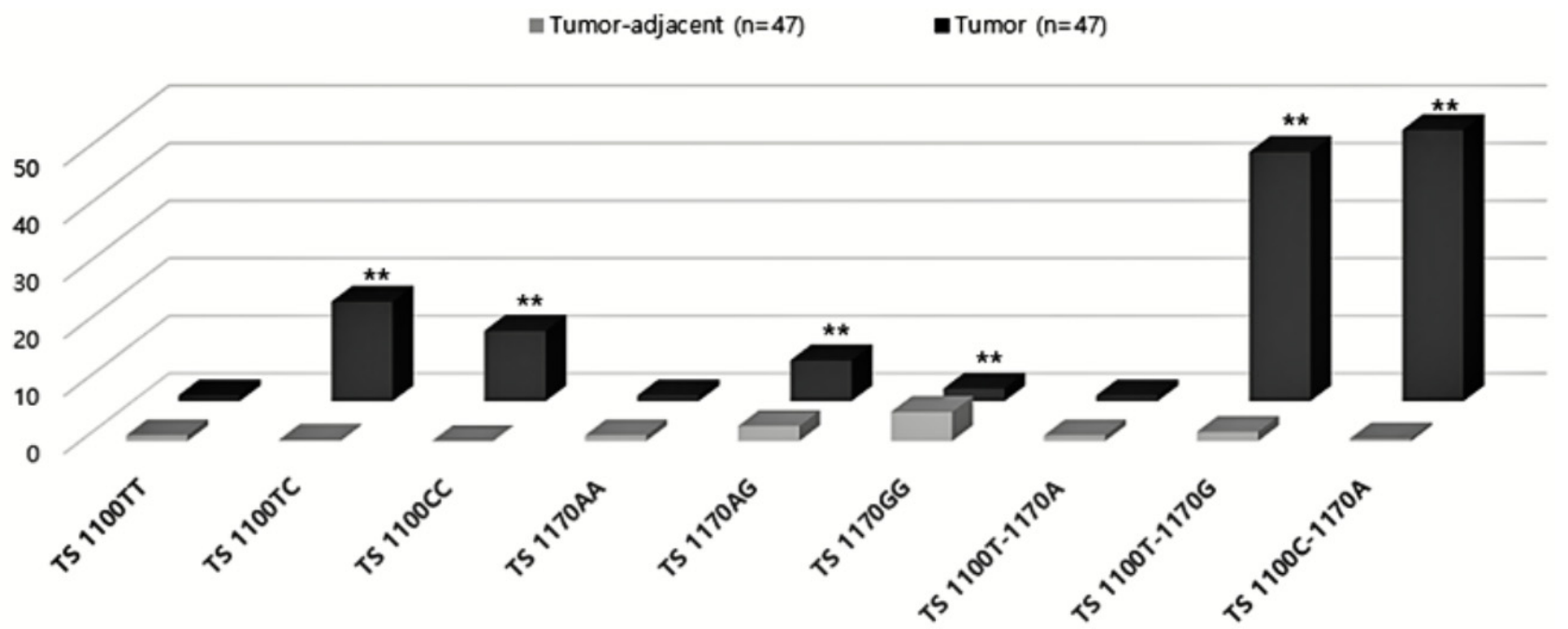

Figure 2. Expression of thymidylate synthase (TS) 1100T $>C$ (rs699517) and 1170A $>$ G(rs2790). Single nucleotide polymorphisms (rs699517 and rs2790) in the $3^{\prime}$-untranslated region (UTR) of TS. Quantitative reverse transcription PCR (qRT-PCR) to measure levels of TS 1100T and 1100C, TS 1170A and TS 1170G, TS 1100T-1170G and TS 1100C-1170A expression in CRC tissue. Means and individual values from three biological replicates are shown. Source data are provided in the source Supplemental Table S5. The housekeeping gene GADPH served as the loading control. ${ }^{* *}$ indicates $p<0.05$.

\subsection{Expression of Target miRNAs}

We next quantified expression of miR-203 and miR-124- 1 in tissue samples and determined whether we could detect differences in expression, based on the TS $3^{\prime}$-UTR polymorphisms and haplotypes (Supplemental Table S6). Compared with the wild-type (WT) 1100TT genotype, expression of miR-203 in 1100TC and 1100CC was found to be decreased in tumor tissue. In the case of TS $1170 \mathrm{~A}>\mathrm{G}$, we detected decreased expression of 1170AG and 1170GG relative to 1170AA (WT) in tumors, similar to what we observed for the TS 1100T>C genotype. However, no statistically significant inverse correlation has been identified in the expression between TS and miRNA.

\section{Discussion}

In the present study, we investigated the association between two new $3^{\prime}$-UTR polymorphisms in the TS gene, 1100T>C (rs699517) and 1170A $>\mathrm{G}$ (rs2790), and the occurrence and prognosis of CRC. We found that the combined genotype and haplotype of TS 1100T>C 
and 1170A $>$ G were strongly associated with CRC susceptibility. For associations with prognosis factors (3-year OS and RFS rates), TS 1100T $>C$ was an effective predictor for poor prognosis. We also found that TS 1170A $>$ G genotypes are strongly associated with CRC susceptibility, with an increased risk of CRC observed in those with the mutant 1170G allele compared to the WT 1170A allele. Our data further indicate that the TS 1100TC, TS 1100CC, and TS 1170AG genotypes, and the TS 1100T-1170G and 1100C-1170A haplotypes are associated with elevated TS expression in CRC.

Previous studies have focused on known TYMS polymorphisms in the promoter region, including the TSER 2R/3R repeat and 5'-UTR (rs45445694), as well as a 6-bp in/del of 3'-UTR (TS 1494del6b, (rs16430)) [22]. In particular, TS 3R alleles, which promote elevated TS expression, have been shown to be potent genetic risk factors for increased CRC susceptibility in meta-analyses [34-36]. Critically, an understanding of how elevated levels of TS promote carcinogenic events could also help to explain why TS $3^{\prime}$-UTR polymorphisms may affect CRC occurrence and prognosis. According to a recent report, compared with the rs2790 AA genotype at the 3'-UTR of TS, the rs2790 GG genotype was associated with a significantly higher risk of acute lymphoblastic leukaemia (ALL) [37]. The present study confirmed that the presence of the TS 3'UTR polymorphism (rs151264360) increased the risk of persistence of cervical cancer [38]. Another report shows that the rs2790 AA genotype has a higher risk of death than GA or GG genotypes in Chinese patients with non-small cell lung cancer (NSCLC) [39].

Plasma FA concentrations inversely correlate with Hcy levels in vivo [40], and it has previously been shown that elevated TS expression can promote increased Hcy and decreased FA levels, leading to induction of CRC development [41,42]. In particular, depletion of FA is thought to promote colorectal carcinogenesis via the induction of DNA breaks in the chromosome [43]. There are plausible mechanisms by which FA deficiency may create such breaks, including uracil misincorporation and impaired DNA repair [44,45]. FA deficiency reduces deoxythymidylate synthesis from deoxyuridylate, and the resulting nucleotide imbalance accelerates the incorporation of uracil bases into DNA. Uracil in DNA is excised by a repair glycosylase, and in the process, a transient single-strand break is generated in the chromosome [46]. FA supplementation has been proposed as a possible solution, however, animal studies have shown that this may increase CRC risk and accelerate CRC progression if too much is given, or if it is provided after neoplastic foci are established in the colorectum $[47,48]$. CRC incidence has been influenced by a variety of risk factors. Therefore, stratified analyses were useful in elucidating CRC epidemiology resulting from a diversity of confounding variables. Our data show that common subsetspecific associations for 1100TC-1170AG, 1100CC-1170AA and TS 1100TT-1170GG were found for the gender, TNM I or II stage, HTN, Hcy and FA subgroups; TS 1100C-1170A haplotypes displayed common subset-specific associations with the age, gender (male), tumor size, TNM I or II stage, HTN, DM, BMI $\left(<25 \mathrm{~kg} / \mathrm{m}^{2}\right), \mathrm{HDL}-\mathrm{C} \geq 40$ (male)/50 (female) $\mathrm{mg} / \mathrm{dL}$, Hcy $(\geq 11.7 \mu \mathrm{mol} / \mathrm{L})$ and FA $(\geq 4.58 \mathrm{ng} / \mathrm{mL})$ subgroups. Because cancer risk is determined by the complex interplay of genetic and environmental factors, we calculated combined gene-environment effects on CRC susceptibility. Notably, our data show that when compared with TS 1170AA WT, the TS 1170AG+GG genotypes display strong geneenvironment combined effects with HTN and FA $<4.58 \mathrm{ng} / \mathrm{mL}$ on CRC incidence. We did not observe any significant gene-environment combined effects with Hcy concentrations, consistent with previous studies that did not detect a significantly elevated CRC risk with increasing plasma Hcy levels [49,50]. Previous studies on the association between plasma Hcy levels and risk of colorectal neoplasia have further yielded complicated results.

Here, we detected increased TS expression in subjects with the TS 1100TC and CC and the TS 1170AG and GG genotypes, relative to those with the respective WT genotypes. Additionally, we find that the risk of CRC is increased in individuals with the mutant (1100CC, 1170GG) or heterozygous genotypes (1100TC, 1170AG) compared to the WT 1100TT and 1170AA genotype. Notably, elevated TS levels are correlated with a poorer prognosis, and increased amounts of TS in tumors are associated with resistance to 5-FU 
chemotherapy. Our data indicate that expression of the mutant type was significantly increased in CRC for both TS 1100, TS 1170 and haplotypes. 5-Fluorouracil (5-FU) is a widely used chemical agent for treating colorectal cancer [51]; however, resistance to 5FU can decrease its therapeutic efficacy. Previous reports have suggested that miRNAs might represent an effective strategy for preventing resistance and improving treatment outcomes. For example, it was shown that miR-203 inhibition enhances cellular resistance to 5-FU, whereas miR-203 overexpression increases 5-FU sensitivity [52]. Notably, functional experiments further showed that the inhibitory effect of $m i R-203$ on tumor growth under 5 -FU exposure is mediated by downregulation of TS, which increases cellular sensitivity to 5-FU. Another report provided direct evidence that $m i R-192$ and $m i R-215$ also influence sensitivity to 5-FU treatment [53]. These data therefore suggest that miRNAs may be useful biomarkers for predicting 5-FU treatment outcomes. Our data show that miR-203 concentrations inversely correlate with TS 1100T $>C$ and TS 1170A $>$ G levels in tissues. Genetic variation in the $3^{\prime}$-UTR region could affect mRNA stability and translation through an altered miRNA binding affinity. Currently, however, there are no data to directly show that miRNA binding activity is affected by TS 1100 or TS $11703^{\prime}$-UTR polymorphisms.

The reason for the observed decrease in expression of miRNAs involved in the regulation of a drug-target gene in tumor tissue remains unclear. However, a possible explanation can be found in a previous report demonstrating that the loss of the tumor suppressor $\mathrm{PKC} z$ in CRC cells is associated with lower ADAR2 activity. This promotes the loading of miR-200s into extracellular vesicles (EVs), thereby decreasing their intracellular steadystate levels. Loss of this axis induces the epithelial-to-mesenchymal transition (EMT) and increased liver metastases, which can be inhibited in vivo by blocking miR-200 release. Therefore, the PKCz/ADAR2 axis is a critical regulator of CRC metastases through modulation of $m i R-200$ levels [54]. Further studies, however, are needed to determine whether a similar mechanism regulates the steady-state levels of microRNAs. Specifically, it will be necessary to identify the factors that regulate expression of this subset of miRNAs. In addition, it is important to determine how regulation of the $3^{\prime}$-UTR of TS by microRNAs might influence 5-FU treatment, and whether this also affects cell proliferation and cancer progression. Importantly, these studies also have the potential to clinically impact many diseases related to $1 \mathrm{C}$ metabolism.

We could not find differences in miRNA binding activity or pre-miRNA expression of target miRNAs as a function of genotype at these loci. Despite a lack of data, these miRNAs may be important genetic factors in the prevalence and progression of CRC because their expression is altered in some of the genotypes and haplotypes tested here. Further studies are needed to directly test for miRNA binding activity to TS 3'-UTR polymorphisms, to determine the mechanism by which these polymorphisms may influence cellular proliferation and cancer progression.

This study has several limitations. First, the manner in which 3'-UTR polymorphisms in the TS genes affect development of CRC is still unclear. Second, the lack of information regarding additional environmental risk factors in CRC patients remains to be investigated. Lastly, the population of this study was restricted to patients of Korean ethnicity. Although results from our study provide the first evidence for $3^{\prime}$-UTR variants in TS genes as potential biomarkers of CRC prevention and prognosis, a prospective study on a larger cohort of patients is warranted to validate these findings.

In conclusion, this study is the first to identify an association between TS $3^{\prime}$-UTR polymorphisms (rs699517, rs2790) and an increased risk of CRC. We found that TS 1170A>G genotypes, as well as the TS 1100T-1170G and 1100C-1170A haplotypes, are strongly associated with CRC. The TS 1100TC+CC type was associated with a poor survival (OS and RFS) rate. Stratified analyses indicated that subset-specific associations for TS 1100/1170 combined genotypes were found for the age, TNM I or II stage, HTN, and FA subgroups. TS 1100C-1170A haplotypes displayed common subset-specific associations with the age, gender (male), tumor size, TNM I or II stage, HTN, DM, HDL-C and Hcy subgroups. 
Supplementary Materials: The following are available online at https: / www.mdpi.com/article/ 10.3390/jpm11060537/s1, Table S1: Adjusted odds ratios of gene-environment combined effects on CRC incidence. Table S2: Survival analysis of TS 3'-UTR polymorphisms in CRC patients. Table S3: Survival analysis for combined genotypes and haplotypes TS in CRC patients. Table S4: Adjusted hazard ratios of prognosis risk factors on CRC patients according to TS 3'-UTR polymorphisms. Table S5: TS mRNA expression according to 3'-UTR genotypes or haplotypes. Table S6: TS, miR-124-1, and miR-203 expression levels according to TS 3'-UTR polymorphisms.

Author Contributions: Conceptualization, Y.-J.J. and N.-K.K.; methodology, C.-S.R. and H.-S.P.; software, Y.-J.J.; validation, C.-S.R. and E.-J.K.; formal analysis, S.-H.C. and H.-J.A.; investigation, J.-Y.L.; resources, J.-Y.L.; data curation, J.-W.K.; writing—original draft preparation, Y.-J.J.; writingreview and editing, S.-H.C.; visualization, E.-J.K.; supervision, J.-W.K.; project administration, N.-K.K.; funding acquisition, N.-K.K. and S.-H.C. All authors have read and agreed to the published version of the manuscript.

Funding: This study was supported by a National Research Foundation of Korea (NRF) Grant (NRF-2020R1I1A1A01060656, 2018R1D1A1B07047604 \& 2020R1I1A1A01072008), funded by the Korean Government.

Institutional Review Board Statement: The study was conducted according to the guidelines of the Declaration of Helsinki, and approved (IRB No. 2009-08-077) by the Institutional Review Board of CHA Bundang Medical Center, Seongnam, South Korea.

Informed Consent Statement: Not applicable.

Data Availability Statement: The data presented in this study are available on request from the corresponding author.

Conflicts of Interest: The authors declare no conflict of interest.

\section{References}

1. Incidence and Mortality Worldwide IARC CancerBase No. 11; International Agency for Research on Cancer: Lyon, France, 2018.

2. Boyle, P.; Levin, B. Colorectal cancer. In World Cancer Report; International Agency for Research on Cancer: Lyon, France, 2008; pp. 374-378.

3. Giovannucci, E.; Ascherio, A.; Rimm, E.B.; Colditz, G.; Stampfer, M.J.; Willett, W.C. Physical Activity, Obesity, and Risk for Colon Cancer and Adenoma in Men. Ann. Intern. Med. 1995, 122, 327-334. [CrossRef] [PubMed]

4. Terzić, J.; Grivennikov, S.; Karin, E.; Karin, M. Inflammation and Colon Cancer. Gastroenterology 2010, 138, 2101-2114.e5. [CrossRef]

5. Tsoi, K.K.; Pau, C.Y.; Wu, W.K.; Chan, F.K.; Griffiths, S.; Sung, J.J. Cigarette Smoking and the Risk of Colorectal Cancer: A Meta-analysis of Prospective Cohort Studies. Clin. Gastroenterol. Hepatol. 2009, 7, 682-688.e5. [CrossRef] [PubMed]

6. Asai, A.; Konno, M.; Koseki, J.; Taniguchi, M.; Vecchione, A.; Ishii, H. One-carbon metabolism for cancer diagnostic and therapeutic approaches. Cancer Lett. 2020, 470, 141-148. [CrossRef] [PubMed]

7. Kawakami, K.; Omura, K.; Kanehira, E.; Watanabe, Y. Polymorphic tandem repeats in the thymidylate synthase gene is associated with its protein expression in human gastrointestinal cancers. Anticancer. Res. 2000, 19, 3249-3252.

8. Mandola, M.V.; Stoehlmacher, J.; Zhang, W.; Groshen, S.; Yu, M.C.; Iqbal, S.; Lenz, H.-J.; Ladner, R.D. A 6 bp polymorphism in the thymidylate synthase gene causes message instability and is associated with decreased intratumoral TS mRNA levels. Pharmacogenetics 2004, 14, 319-327. [CrossRef] [PubMed]

9. Papamichael, D. The use of thymidylate synthase inhibitors in the treatment of advanced colorectal cancer: Current status. Oncology 1999, 4, 478-487.

10. Berger, F.G.; Berger, S.H. Thymidylate synthase as a chemotherapeutic drug target: Where are we after fifty years? Cancer Biol. Ther. 2006, 5, 1238-1241. [CrossRef] [PubMed]

11. Leichman, L.; Lenz, H.-J.; Leichman, C.; Groshen, S.; Danenberg, K.; Baranda, J.; Spears, C.; Boswell, W.; Silberman, H.; Ortega, A.; et al. Quantitation of intratumoral thymidylate synthase expression predicts for resistance to protracted infusion of 5-fluorouracil and weekly leucovorin in disseminated colorectal cancers: Preliminary report from an ongoing trial. Eur. J. Cancer 1995, 31, 1306-1310. [CrossRef]

12. Johnston, P.G.; Fisher, E.R.; Rockette, H.E.; Fisher, B.; Wolmark, N.; Drake, J.C.; Chabner, B.A.; Allegra, C.J. The role of thymidylate synthase expression in prognosis and outcome of adjuvant chemotherapy in patients with rectal cancer. J. Clin. Oncol. 1994, 12, 2640-2647. [CrossRef]

13. Lenz, H.J.; Hayashi, K.; Salonga, D.; Danenberg, K.D.; Danenberg, P.V.; Metzger, R.; Banerjee, D.; Bertino, J.R.; Groshen, S.; Leichman, L.P.; et al. p53 point mutations and thymidylate synthase messenger RNA levels in disseminated colorectal cancer: An analysis of response and survival. Clin. Cancer Res. 1998, 4, 1243-1250. 
14. Cheng, T.Y.; Makar, K.W.; Neuhouser, M.L.; Miller, J.W.; Song, X.; Brown, E.C.; Beresford, S.A.; Zheng, Y.; Poole, E.M.; Galbraith, R.L.; et al. Folate-mediated one-carbon metabolism genes and interactions with nutritional factors on colorectal cancer risk: Women's Health Initiative Observational Study. Cancer 2015, 15, 3684-3691. [CrossRef]

15. Haerian, B.S.; Haerian, M.S. Evaluation of association studies and meta-analyses of MTHFR gene polymorphisms in colorectal cancer. Pharmacogenomics 2015, 16, 413-425. [CrossRef]

16. Jeon, Y.J.; Kim, J.W.; Park, H.M.; Kim, J.O.; Jang, H.G.; Oh, J.; Hwang, S.G.; Kwon, S.W.; Oh, D.; Kim, N.K. Genetic variants in 3'-UTRs of methylenetetrahydrofolate reductase (MTHFR) predict olorectal cancer susceptibility in Koreans. Sci. Rep. 2015, 5, 11006. [CrossRef]

17. Ose, J.; Botma, A.; Balavarca, Y.; Buck, K.; Scherer, D.; Habermann, N.; Beyerle, J.; Pfütze, K.; Seibold, P.; Kap, E.J.; et al. Pathway analysis of genetic variants in folate-mediated one-carbon metabolism-related genes and survival in a prospectively followed cohort of colorectal cancer patients. Cancer Med. 2018, 7, 2797-2807. [CrossRef] [PubMed]

18. Nelson, B.; Carter, J.V.; Eichenberger, M.R.; Netz, U.; Galandiuk, S. Genetic polymorphisms in 5-Fluorouracil-related enzymes predict pathologic response after neoadjuvant chemoradiation for rectal cancer. Surgery 2016, 160, 1326-1332. [CrossRef]

19. Horie, N.; Aiba, H.; Oguro, K.; Hojo, H.; Takeishi, K. Functional Analysis and DNA Polymorphism of the Tandemly Repeated Sequences in the 5'-terminal Regulatory Region of the Human Gene for Thymidylate Synthase. Cell Struct. Funct. 1995, 20, 191-197. [CrossRef] [PubMed]

20. Marsh, S.; Collie-Duguid, E.S.; Li, T.; Liu, X.; McLeod, H.L. Ethnic Variation in the Thymidylate Synthase Enhancer Region Polymorphism among Caucasian and Asian Populations. Genomics 1999, 58, 310-312. [CrossRef] [PubMed]

21. Ulrich, C.M.; Bigler, J.; Velicer, C.M.; Greene, E.A.; Farin, F.M.; Potter, J.D. Searching expressed sequence tag databases: Discovery and confirmation of a common polymorphism in the thymidylate synthase gene. Cancer Epidemiol. Biomark. Prev. 2000, 9, 1381-1385.

22. Pullarkat, S.; Stoehlmacher, J.; Ghaderi, V.S.; Xiong, Y.-P.; Ingles, S.A.; Sherrod, A.; Warren, R.M.; Tsaowei, D.D.; Groshen, S.; Lenz, H.-J. Thymidylate synthase gene polymorphism determines response and toxicity of 5-FU chemotherapy. Pharmacogenomics $\mathrm{J}$. 2001, 1, 65-70. [CrossRef]

23. Mauritz, R.; Giovannetti, E.; Beumer, I.J.; Smid, K.; Van Groeningen, C.J.; Pinedo, H.M.; Peters, G.J. Polymorphisms in the enhancer region of the thymidylate synthase gene are associated with thymidylate synthase levels in normal tissues but not in malignant tissues of patients with colorectal cancer. Clin. Colorectal Cancer 2009, 146-154. [CrossRef] [PubMed]

24. Dotor, E.; Cuatrecases, M.; Martínez-Iniesta, M.; Navarro, M.; Vilardell, F.; Guinó, E.; Pareja, L.; Figueras, A.; Mollevi, D.G.; Serrano, T.; et al. Tumor Thymidylate Synthase 1494del6 Genotype As a Prognostic Factor in Colorectal Cancer Patients Receiving Fluorouracil-Based Adjuvant Treatment. J. Clin. Oncol. 2006, 24, 1603-1611. [CrossRef]

25. Figueiredo, J.C.; Levine, A.J.; Crott, J.W.; Baurley, J.; Haile, R.W. Folate-genetics and colorectal neoplasia: What we know and need to know next. Mol. Nutr. Food Res. 2013, 57, 607-627. [CrossRef]

26. Ambros, V. The functions of animal microRNAs. Nature 2004, 431, 350-355. [CrossRef] [PubMed]

27. Bartel, D.P. MicroRNAs: Genomics, Biogenesis, Mechanism, and Function. Cell 2004, 116, 281-297. [CrossRef]

28. Yang, Y.; Zhang, Q.; Miao, Y.-R.; Yang, J.; Yang, W.; Yu, F.; Wang, D.; Guo, A.-Y.; Gong, J. SNP2APA: A database for evaluating effects of genetic variants on alternative polyadenylation in human cancers. Nucleic Acids Res. 2019, 48, D226-D232. [CrossRef]

29. Charles, S.; Natarajan, J. Two way network construction and analysis of mRNA, miRNA and lncRNA reveals critical regulators and regulatory modules in cardiovascular diseases. Genes Genom. 2020, 42, 855-867. [CrossRef] [PubMed]

30. Giovannucci, E. Metabolic syndrome, hyperinsulinemia, and colon cancer: A review. Am. J. Clin. Nutr. 2007, 86, 836S-842S. [CrossRef] [PubMed]

31. Ritchie, M.D.; Hahn, L.W.; Roodi, N.; Bailey, L.R.; Dupont, W.D.; Parl, F.F.; Moore, J.H. Multifactor-Dimensionality Reduction Reveals High-Order Interactions among Estrogen-Metabolism Genes in Sporadic Breast Cancer. Am. J. Hum. Genet. 2001, 69, 138-147. [CrossRef]

32. Moore, J.H.; Williams, S.M. New strategies for identifying gene-gene interactions in hypertension. Ann. Med. 2002, 34, 88-95. [CrossRef] [PubMed]

33. Lee, S.Y.; Chung, Y.; Elston, R.C.; Kim, Y.; Park, T. Log-linear model-based multifactor dimensionality reduction method to detect gene gene interactions. Bioinformatics 2007, 23, 2589-2595. [CrossRef]

34. Lu, M.; Sun, L.; Yang, J.; Li, Y.-Y. 3R variant of thymidylate synthase 5 '-untranslated enhanced region contributes to colorectal cancer risk: A meta-analysis. Asian Pac. J. Cancer Prev. 2012, 13, 2605-2610. [CrossRef]

35. Teng, Z.; Wang, L.; Cai, S.; Yu, P.; Wang, J.; Gong, J.; Liu, Y. The 677C >T (rs1801133) Polymorphism in the MTHFR Gene Contributes to Colorectal Cancer Risk: A Meta-Analysis Based on 71 Research Studies. PLoS ONE 2013, 8, e55332. [CrossRef]

36. Subbarayan, P.R.; Hammad, H.; Sarkar, M.; Ardalan, B.; Gupta, N. The presence of three repeats in the $5^{\prime}$ UTR region of thymidylate synthase (TS) is associated with increased TS mRNA expression in cultured human cancer cell lines in vitro. Oncol. Rep. 2011, 27, 246-249. [CrossRef]

37. Wang, S.-M.; Zeng, W.-X.; Wu, W.-S.; Sun, L.-L.; Yan, D. Genotype and allele frequencies of TYMS rs2790 A > G polymorphism in a Chinese paediatric population with acute lymphoblastic leukaemia. J. Clin. Pharm. Ther. 2018, 43, 507-512. [CrossRef] [PubMed]

38. Silva, N.N.T.; Santos, A.C.S.; Nogueira, V.M.; Carneiro, C.M.; Lima, A.A. 3'UTR polymorphism of Thymidylate Synthase gene increased the risk of persistence of pre-neoplastic cervical lesions. BMC Cancer 2020, 20, 323. [CrossRef] [PubMed] 
39. Xu, J.; Tian, S.; Yin, Z.; Wu, S.; Liu, L.; Qian, Y.; Pei, D.; Gao, W.; Xu, J.; Yinet, Y.; et al. MicroRNA-binding site SNPs in deregulated genes are associated with clinical outcome of non-small cell lung cancer. Lung Cancer 2014, 85, 442-448. [CrossRef] [PubMed]

40. Chen, F.-P.; Lin, C.-C.; Chen, T.-H.; Tsai, M.-C.; Huang, Y.-C. Higher Plasma Homocysteine Is Associated with Increased Risk of Developing Colorectal Polyps. Nutr. Cancer 2013, 65, 195-201. [CrossRef] [PubMed]

41. Miller, J.W.; Beresford, S.A.A.; Neuhouser, M.L.; Cheng, T.-Y.D.; Song, X.; Brown, E.C.; Zheng, Y.; Rodriguez, B.; Green, R.; Ulrich, C.M. Homocysteine, cysteine, and risk of incident colorectal cancer in the Women's Health Initiative observational cohort. Am. J. Clin. Nutr. 2013, 97, 827-834. [CrossRef]

42. Brevik, A.; Joshi, A.D.; Corral, R.; Onland-Moret, N.C.; Siegmund, K.D.; Le Marchand, L.; Baron, J.A.; Martinez, M.E.; Haile, R.W.; Ahnen, D.J.; et al. Polymorphisms in Base Excision Repair Genes as Colorectal Cancer Risk Factors and Modifiers of the Effect of Diets High in Red Meat. Cancer Epidemiol. Biomark. Prev. 2010, 19, 3167-3173. [CrossRef]

43. Branda, R.F.; Blickensderfer, D.B. Folate deficiency increases genetic damage caused by alkylating agents and gamma-irradiation in Chinese hamster ovary cells. Cancer Res. 1993, 53, 5401-5408.

44. Choi, S.-W.; Kim, Y.-I.; Weitzel, J.N.; Mason, J.B. Folate depletion impairs DNA excision repair in the colon of the rat. Gut 1998, 43, 93-99. [CrossRef]

45. Duthie, S.J.; Narayanan, S.; Blum, S.; Pirie, L.; Brand, G.M. Folate Deficiency In Vitro Induces Uracil Misincorporation and DNA Hypomethylation and Inhibits DNA Excision Repair in Immortalized Normal Human Colon Epithelial Cells. Nutr. Cancer 2000, 37, 245-251. [CrossRef] [PubMed]

46. Blount, B.C.; Mack, M.M.; Wehr, C.M.; MacGregor, J.T.; Hiatt, R.A.; Wang, G.; Wickramasinghe, S.N.; Everson, R.B.; Ames, B.N. Folate deficiency causes uracil misincorporation into human DNA and chromosome breakage: Implications for cancer and neuronal damage. Proc. Natl. Acad. Sci. USA 1997, 94, 3290-3295. [CrossRef] [PubMed]

47. Biasco, G.; Zannoni, U.; Paganelli, G.M.; Santucci, R.; Gionchetti, P.; Rivolta, G.; Miniero, R.; Pironi, L.; Calabrese, C.; Di Febo, G.; et al. Folic acid supplementation and cell kinetics of rectal mucosa in patients with ulcerative colitis. Cancer Epidemiol. Biomark. Prev. 1997, 6, 469-471.

48. Akoglu, B.; Faust, D.; Milovic, V.; Stein, J. Folate and chemoprevention of colorectal cancer: Is 5-methyl-tetrahydrofolate an active antiproliferative agent in folate-treated colon-cancer cells? Nutriens 2001, 17, 652-653. [CrossRef]

49. van der Put, N.M.; Gabreëls, F.; Stevens, E.M.; Smeitink, J.A.; Trijbels, F.J.; Eskes, T.K.; Heuvel, L.P.V.D.; Blom, H. A Second Common Mutation in the Methylenetetrahydrofolate Reductase Gene: An Additional Risk Factor for Neural-Tube Defects? Am. J. Hum. Genet. 1998, 62, 1044-1051. [CrossRef]

50. Weinstein, S.J.; Albanes, D.; Selhub, J.; Graubard, B.; Lim, U.; Taylor, P.R.; Virtamo, J.; Stolzenberg-Solomon, R. One-Carbon Metabolism Biomarkers and Risk of Colon and Rectal Cancers. Cancer Epidemiol. Biomark. Prev. 2008, 17, 3233-3240. [CrossRef]

51. Longley, D.B.; Harkin, D.P.; Johnston, P.G. 5-Fluorouracil: Mechanisms of action and clinical strategies. Nat. Rev. Cancer 2003, 3, 330-338. [CrossRef] [PubMed]

52. Li, T.; Gao, F.; Zhang, X.-P. miR-203 enhances chemosensitivity to 5-fluorouracil by targeting thymidylate synthase in colorectal cancer. Oncol. Rep. 2014, 33, 607-614. [CrossRef]

53. Boni, N.; Bitarte, I.; Cristobal, R.; Zarate, J.; Rodriguez, E.; Maiello, J.; Garcia-Foncillas, J.; Bandres, E. miR-192/miR-215 influence 5-fluorouracil resistance through cell cycle-mediated mechanisms complementary to its post-transcriptional thymidilate synthase regulation. Mol. Cancer. Ther. 2010, 9, 2265-2275. [CrossRef] [PubMed]

54. Shelton, P.M.; Duran, A.; Nakanishi, Y.; Reina-Campos, M.; Kasashima, H.; Llado, V.; Ma, L.; Campos, A.; Olmo, D.G.; GarcíaArranz, M.; et al. The Secretion of miR-200s by a PKC C/ADAR2 Signaling Axis Promotes Liver Metastasis in Colorectal Cancer. Cell Rep. 2018, 23, 1178-1191. [CrossRef] [PubMed] 